\title{
Current perspectives on tinnitus
}

\section{M Baguley, D J McFerran}

\section{Experience of tinnitus is common in childhood}

T innitus in adults has received much scientific and clinical attention of late. Traditional theories about the pathogenesis of tinnitus concentrated on the cochlea, but this model had some serious shortcomings and could not explain some clinical findings. A purely cochlear model of tinnitus fails to explain those patients who have tinnitus in the presence of normal hearing and conversely why many patients with cochlear hearing loss do not have tinnitus. In addition, symptoms of tinnitus related anxiety, insomnia, and general agitation are very common in adults troubled with tinnitus ${ }^{1}$ and cannot be ascribed to cochlear dysfunction alone.

To explain these seeming paradoxes it is necessary to look at what happens to auditory information in the brain as well as in the ear. Information is sent from the cochlea to the brain stem and then to the primary and associative auditory cortices in the temporal lobes to give the conscious sensation of hearing. However, information is also sent from brain stem auditory nuclei to the reticular formation and to the sympathetic autonomic nervous system. These systems are involved with reaction to alerting or challenging stimuli. In addition information is sent to the limbic system, specifically the amygdalae, which are involved in emotional responses. These connections are vital for the role of hearing as the major alerting and warning sense, and a consensus view that the mammalian auditory system evolved to allow the perception of sounds with survival value is now held. ${ }^{2}$ If there is an audible threat in the environment the brain filters that threat from other auditory information and instigates an appropriate limbic and reticular reaction with a sympathetically mediated fight or flight response. Tinnitus distress arises when this response is generated not to an outside stimulus but to internal neural signals. The auditory system is not an electrically quiet system and there are many potential tinnitus generating internal signals at both peripheral and central levels. These theories were united in a neurophysiological model of tinnitus by Jastreboff in 1990. ${ }^{3}$ Treatments deriving from this model have been described, with the aim of facilitating habituation to tinnitus. In particular, tinnitus retraining therapy ${ }^{4}$ has been advocated, being a combination of explaining the nature and pathogenesis of the condition, reassurance, counselling, and sound therapy. Initial work to determine the efficacy of this approach showed promise, ${ }^{5}$ and randomised controlled trials are now underway.

Tinnitus in childhood has not received the same amount of attention as tinnitus in adulthood despite the fact that a complaint of persistent tinnitus in a child presents a significant therapeutic challenge. This dearth of interest is in part a result of the fact that children rarely present clinically with tinnitus. However, when children are specifically asked about the symptom a substantial proportion have tinnitus. To understand how childhood tinnitus should be managed it is necessary to examine the prevalence and incidence of tinnitus in childhood, to consider how children who complain of tinnitus should be investigated, and to decide how tinnitus treatment strategies should be applied to this group.

\section{TINNITUS IN NORMALLY HEARING CHILDREN}

Research into the prevalence of tinnitus in children is difficult as once specific questions are asked there is a risk that the child may try to please their adult questioner and in some studies there may be overestimates. An additional issue in interpretation of the literature is that there is no consensus in international tinnitus research as to what constitutes a child, thus in the studies cited below different age ranges are examined and the results may not be comparable.

In a large well conducted population survey, $10 \%$ of adults questioned reported prolonged ( $>5$ minutes) spontaneous tinnitus. ${ }^{6}$ Accepting the limitations of studies in children, tinnitus prevalence childhood shows a not dissimilar picture. Nodar, ${ }^{7}$ in a survey of 2000 normally hearing children (aged 1 1-18 years) found a tinnitus prevalence of $15 \%$, though no study was made of severity. Mills et al questioned 93 normally hearing children (aged 5-16 years, mean age 5.7 years) about tinnitus. ${ }^{8}$ Of these, $27(29 \%)$ reported tinnitus, and nine $(9.7 \%)$ stated that they were "bothered" by their tinnitus. The authors felt that this prevalence of tinnitus might have been an underestimate because of difficulties in communicating with the children. A recent study ${ }^{9}$ considered the prevalence of tinnitus in a group of 7 year old children in Sweden $(n=120)$; a prevalence of $12 \%$ was determined, rising to $13 \%$ if only normally hearing children were considered.

The question of the validity of tinnitus prevalence studies in children was addressed by Stouffer and colleagues. ${ }^{10}$ This study attempted to determine the prevalence of tinnitus in normally hearing and hearing impaired children, utilising a test method that also measured the consistency of the child's responses. Depending on the level of consistency of response required, the findings were that $6-13 \%$ of normal hearing children and $24-29 \%$ of hearing impaired children reported tinnitus of duration $>5$ minutes. This is the only study in this field that takes account of the effect of children's desire to please adult investigators by agreeing with them.

\section{TINNITUS IN CHILDREN WITH A SIGNIFICANT SENSORINEURAL HEARING LOSS (SNHL)}

Several studies have investigated the prevalence of tinnitus in children with SNHL. Table 1 summarises the results. While there are variations in the methodology of these studies, specifically regarding the age of children studied and the extent of the SNHL, there are indications that:

- The prevalence of tinnitus in children with sensorineural hearing loss appears greater than that in normally hearing children

- The prevalence of tinnitus in children with profound SNHL appears lower than in those children with moderate/ severe SNHL

- An acquired SNHL is more likely to be associated with tinnitus than a congenital loss

- It is rare for a child with SNHL to spontaneously complain of tinnitus.

\section{TINNITUS IN CHILDREN WITH} OTHER OTOLOGICAL DISORDERS

Mills and Cherry ${ }^{16}$ report a series of 66 children (aged 5-15 years) presenting to an ear, nose, and throat outpatient facility with conductive hearing losses caused by secretory otitis media. In this series 29 children (43.9\%) reported tinnitus compared to a control group of 44 children with SNHL, in whom 13 $(29.5 \%)$ reported tinnitus. The prevalence of tinnitus in the normally hearing group was $29 \%$.

Mills et al considered a group of 403 children (aged 5-15 years) seen by two otologists. ${ }^{8}$ Of these, 267 (66\%) were said to have evidence of ear disease while the 
Table 1 Studies of tinnitus prevalence in shildren with SNHL

\begin{tabular}{|c|c|c|c|c|}
\hline Author & $\mathrm{n}$ & Ages & $\begin{array}{l}\text { Prevalence } \\
\text { of tinnitus }\end{array}$ & Notes \\
\hline Nodar and Lezak" & 55 & $11-18$ & $56 \%$ & $\begin{array}{l}\text { Prevalence in moderate loss } 100 \% \\
\text { Prevalence in profound loss } 35 \%\end{array}$ \\
\hline Reich $^{12}$ & 46 & & $39 \%$ & Quoted in Graham ${ }^{13}$ \\
\hline Graham $^{13}$ & 158 & $12-18$ & $49 \%$ & $\begin{array}{l}\text { Prevalence in moderate/severe } 66 \% \\
\text { Prevalence in profound } 29 \%\end{array}$ \\
\hline Viani $^{14}$ & 102 & $6-17$ & $23.5 \%$ & $\begin{array}{l}\text { Only } 3 \text { children had previously complained of tinnitus } \\
\text { Mostly profound losses }\end{array}$ \\
\hline Drukier $^{15}$ & 331 & & $33 \%$ & All profound \\
\hline Stouffer et al ${ }^{10}$ & 21 & $7-10$ & $24-29 \%$ & $\begin{array}{l}\text { Consistency of response determined } \\
\text { Hearing loss not detailed }\end{array}$ \\
\hline
\end{tabular}

remainder did not: this "ear disease" was in the main SNHL or secretory otitis media. There was said to be a statistically significant difference between the prevalence of tinnitus in the ear disease group but figures are not clearly given. Thus it is difficult to make an inference about the role of secretory otitis media in the tinnitus experience in children.

Because it is rare for a child to complain spontaneously of tinnitus, it is important to consider the characteristics of those who do. Martin and Snashall ${ }^{17}$ considered children who had presented to departments of audiological medicine with a complaint of tinnitus in five centres in the UK. In this series of 67 cases, 42 returned a questionnaire. Of these, 35 children reported bothersome tinnitus $(83 \%)$. In the total series of 67 , a diagnosis of migraine was made in 13 cases $(19 \%)$, and features suggestive of juvenile Meniere's disease were present in five $(7 \%)$. Other diagnoses included endolymphatic hydrops (three cases), chronic suppurative otitis media (three cases), noise induced hearing loss (three cases), and one case of a brain stem tumour. Other workers have also reported a small incidence of more serious otological pathology in childhood that has tinnitus as a symptom. Additional cases of juvenile Meniere's disease have been reported, ${ }^{18}{ }^{19}$ as has perilymph fistula in childhood. ${ }^{19} 20$ The youngest case of vestibular schwannoma in a large series was aged 13 years ${ }^{21}$ : in such cases neurofibromatosis II is a more likely diagnosis than sporadic vestibular schwannoma. ${ }^{22}$ Thus the possibility of treatable and potentially serious pathology in childhood tinnitus should be borne in mind.

\section{HYPERACUSIS}

Abnormal sensitivity to sound of moderate intensity, or hyperacusis has been noted in $40 \%$ of adults with distressing tinnitus. ${ }^{4}$ Recent advances in the understanding of hearing physiology have shown that information in the auditory pathways does not flow just from the ear to the brain: there is also considerable flow in the opposite direction from brain to ear. This efferent or descending auditory system is hypothesised to have a major role in mediating the gain of the human auditory system. ${ }^{23}$ Dysfunction of the efferent system has been suggested as a cause of hyperacusis, ${ }^{24}$ as has disturbance of 5-HT pathways in the brain. ${ }^{25}$ There are no well conducted studies of the prevalence of hyperacusis in children in the literature but anecdotally it seems quite common. The common initial reaction to hyperacusis is to try and avoid the sounds that the person finds uncomfortable, and in adults and older children this may result in recourse to earplugs. This is unhelpful, as if sound above a certain intensity is avoided the auditory system reacts by increasing its sensitivity. Thus an even quieter sound now seems unpleasantly loud and the problem escalates. The appropriate management of hyperacusis is a gradual, controlled reintroduction of sound, facilitating desensitisation of the auditory system.

\section{TINNITUS MANAGEMENT IN CHILDHOOD}

There have been no controlled trials determining the efficacy of tinnitus management strategies in childhood. There is also a question as to whether any intervention is needed: many children do not find the experience of tinnitus distressing, and in many of those who do the symptom resolves with time. Most adults with severe tinnitus develop their tinnitus in adulthood and were not childhood tinnitus sufferers. However, for the small number of children who present with significant tinnitus, some form of help is undeniably required.

It is suggested that a child with a persistent complaint of distressing tinnitus should undergo a consultation in a multidisciplinary paediatric otology clinic. Such clinics exist in most departments of otolaryngology, and although the personnel in the clinic vary from unit to unit, there are generally medical and audiological staff, together with speech and language therapists and teachers of the deaf. A full otological examination is indicated, as is pure tone audiometry and tympanometry. Tinnitus pitch and intensity matching have not been shown to be predictive of treatment outcome in adults, but may have some therapeutic value in allowing parents to listen to a match of their child's tinnitus. History taking should be careful to elicit information about the onset of tinnitus, any associated negative life events, and behavioural or emotional difficulties related to the tinnitus.

The initial history, examination, and simple audiometric tests will determine whether any more involved investigations or specific treatments are required. When this phase is complete, tinnitus therapy can be instituted. Care must be taken to discuss the model of tinnitus in age appropriate language. In many cases the parents will be as anxious and distressed as the child. This should be openly discussed in the presence of an older child, though younger children sometimes find this upsetting. Sound therapy is justified where there is evidence of significant distress, the objective of sound therapy for tinnitus being to reduce the contrast of tinnitus against the background. This can be accomplished by the use of wide band noise generators worn in the ear, though care should be taken to make these cosmetically acceptable and ensure that they do not occlude the ear canal: occluding the external auditory meatus would cause a conductive hearing loss which would be counterproductive. Alternatively environmental sound generators can be used. These may be custom built devices producing repetitive non-intrusive boring sounds or may be something as simple and prosaic as a reassuring ticking 
clock. In children with a hearing impairment, the use of hearing aids is indicated. This helps to reduce the sensitivity of the auditory system by reducing the need to strain to hear. This in turn reduces the awareness of internally generated signals in the auditory pathways. In any case, quite separately from the tinnitus issue, there is robust evidence that even a mild unilateral hearing loss has adverse educational consequences if uncorrected. ${ }^{26}$

Kentish et al noted that sleep disturbance was the most commonly reported concern in a group of 24 children reviewed, having been referred to a department of psychology for tinnitus management. ${ }^{27}$ This finding is congruent with findings in adult tinnitus patients. Care should therefore be taken to issue appropriate devices such as bedside wide band noise generators in this group.

In cases where tinnitus distress appears to have been precipitated by psychological distress, for example as a result of bullying or of bereavement, or where tinnitus is associated with severe emotional distress, one should call upon the support of child psychologist colleagues. In the authors' experience such colleagues will avow ignorance of tinnitus, and joint management is indicated.

\section{CONCLUSION}

Experience of tinnitus appears common in childhood, as in adults, but remarkably few children seem to be upset by this experience. In a small number of children, however, there is sufficient distress associated with tinnitus to necessitate careful management. A diagnostic approach involving the multidisciplinary paediatric otology team is advocated. The therapeutic approach should adopt the same framework as for adults, but using age appropriate language and involving the parents as well as the child.

\section{ACKNOWLEDGEMENT}

Tinnitus research in Cambridge is supported in part by the British Tinnitus Association, www.tinnitus.org.uk

Arch Dis Child 2002;86:141-143

\section{Authors' affiliations}

D M Baguley, Audiology, Addenbrooke's Hospital, Hills Road, Cambridge CB2 2QQ, UK D J McFerran, Essex County Hospital, Lexden Road, Colchester, Essex CO3 3NB, UK

Correspondence to: $\operatorname{Dr} D$ M Baguley; dmb29@cam.ac.uk

\section{REFERENCES}

1 Tyler RS, Baker L Difficulties experienced by tinnitus sufferers. J Speech Lang Res 1983;8:150-4

2 Eggermont JJ. Between sound and perception: reviewing the search for a neural code. Hear Res 200 1;157: 1-42.

3 Jastreboff PJ. Phantom auditory perception (tinnitus): mechanisms of generation and perception. Neurosci Res 1990;8:221-54.

4 Jastreboff PJ, Gray WC, Gold SL. Neurophysiological approach to tinnitus patients. Am J Otol 1996;17:236-40.

5 McKinney CJ, Hazell JWP, Graham RL. An evaluation of the TRT method. In: Hazell JWP, ed. Proceedings of Sixth International Tinnitus Seminar. London: Tinnitus and Hyperacusis Centre, 1999:99-105

6 Davis A, El-Refaie EA. Epidemiology of tinnitus. In: Tyler RS, ed. Tinnitus handbook. San Diego: Singular, 2000.

7 Nodar RH. Tinnitus aurium in school age children. J Aud Res 1972;12:133-5. 8 Mills RP, Albert DM, Brain CE. Tinnitus in childhood. Clin Otolaryngol 1986;11:431-4.

9 Holgers KM. Tinnitus in 7-year-old children. In: Hazell JWP, ed. Proceedings of Vlth International Tinnitus Seminar. London, 1999:218-19.

10 Stouffer JL, Tyler RS, Booth JC, Buckrell B. Tinnitus in normal-hearing and hearing-impaired children. In: Aran JM,
Dauman R, eds. Proceedings of Fourth International Tinnitus Seminar. Kugler, 1992:255-8.

11 Nodar RH, Lezak MHW. Paediatric tinnitus: a thesis revisited. J Laryngol Otol 1984(suppl 9):234-5.

12 Reich G. Personal communication. Cited in Graham JM. Tinnitus in hearing-impaired children. In: Hazell JWP, ed. Tinnitus. London: Churchill Livingstone, 1987:131,143

13 Graham JM. Tinnitus in hearing-impaired children. In: Hazell JWP, ed. Tinnitus. London: Churchill Livingstone, 1987:131,143.

14 Viani LG. Tinnitus in children with hearing loss. J Laryngol Otol 1989;103:142-5.

15 Drukier GS. The prevalence and characteristics of tinnitus with profound sensorineural hearing impairment. Am Ann Deaf 1989;134:260-4.

16 Mills RP, Cherry JR. Subjective tinnitus in children with otological disorders. Int J Paediatr Otorhinolaryngol 1984;7:21-7.

17 Martin K, Snashall S. Children presenting with tinnitus: a retrospective study. $\mathrm{Br} J$ Audio 1994;28:111-15.

18 Hausler R, Toupet M, Cuidetti G, et al. Meniere's disease in children. Am J Otolaryngol 1987:8:187-93.

19 Telischi FF, Rodgers GK, Balkany TJ. Dizziness in childhood. In: Jackler RK, Brackmann DE, eds. Neurotology. New York: Mosby Year Book, 1994:555-66.

20 Parnes LS, McCabe BF. Perilymph fistula: an important cause of deafness and dizziness in children. Paediatrics 1989:80:524-8.

21 Moffat DA, Baguley DM, Beynon GJ, Da-Cruz $M$. Clinical acumen and vestibular schwannoma. Am J Otol 1998;19:82-7.

22 Chang P, Moffat DA. Neurofibromatosis type 2: a 15 year experience-a current review. Aust J Otolaryngol 2000;3:528-42.

23 Sahey TL, Nodar RH, Musiek FE. Efferent auditory system. San Diego: Singular, 1997.

24 Jastreboff PJ, Hazell, JWP. A neurophysiological approach to tinnitus: clinical implications. Br J Audiol clinical implication

25 Marriage J, Barnes NM. Is central hyperacusis a symptom of 5-hydroxytryptamine (5-HT) dysfunction? Laryngol Otol 1995;109:915-21.

26 Bess FH, Dodd-Murphy J, Parker RA. Children with minimal sensorineural hearing loss: prevalence, educational performance, and functional status. Ear Hear 1998; 19:339-54

27 Kentish RC, Crocker SR, McKenna L. Children's experience of tinnitus: a preliminary survey of children presenting to a psychology department. Br J Audiol 2000:34:335-40 